\title{
Compact HTS Cryogen-Free Magnet for Magneto-Optics Research Setup
}

\author{
Y. Wolfus, A. Friedman, F. Kopansky, Y. Yeshurun, Z. Bar-Haim, Z. Ron, and N. Pundak
}

\begin{abstract}
Small working distances between the objective lenses and the inspected samples in magneto-optical (MO) setups limit the volume available for external magnetic field generation coils. Air or water-cooled copper coils are commonly used for field generation, however these coils usually make it possible to attain limited fields of the order of 10-50 mT, in continuous operating mode. HTS coils offer a unique and cost effective solution in such cases of limited space. A compact, $0.4 \mathrm{~T}$, cryogen-free, HTS magnet has been designed, built and tested in a MO setup. The magnet is made of 3,100 turns single pancakes, mounted on the second stage of a two-stage cryocooler. The HTS coil operates at about $\mathrm{T}=45 \mathrm{~K}$ and generates $0.4 \mathrm{~T}$ in a warm bore of $30 \mathrm{~mm}$ diameter. Minimization of metal components in the coil makes it possible to attain field ramp rates of $7.4 \mathrm{~T} / \mathrm{sec}$ with a coil charging voltage of $20 \mathrm{~V}$. Initial cool-down time of the magnet takes less than 5 hours. The magnet design and performances are described in detail and serve as an example for solutions that HTS technology may offer in limited space applications.
\end{abstract}

Index Terms-BSCCO tape, cryogen-free magnet, HTS coil, magneto-optical setup.

\section{INTRODUCTION}

$\mathbf{R}$ ECENTLY, industrial scale manufacturing of HTS Bi-2223 tape began by several companies around the world [1]-[3]. Tapes performances show an on going amelioration, and the price has a strong decrease tendency. A parallel fast progress in cryocoolers technology provides the necessary prerequisites for the development of cryogen-free HTS magnet systems for use in various applications. In such systems, the use of Bi-2223 tapes offers a technologically convenient temperature range for operation, namely $20-40 \mathrm{~K}$. Also, the cryogenic design is relatively simplified because of the use of HTS current leads that substantially decrease the heat input into the cold volume. On the user side, cryogen-free superconducting magnets are attractive because almost no maintenance is required and the operating costs are relatively low.

At present, the technology for making cryogen-free HTS magnets is available and proven [4]-[6]. However, commercialization of HTS magnets depends on economic considerations. The high HTS tapes cost in comparison with LTS wires prevent the commercialization of large magnets. On the other extreme, it is hard to compete with resistive magnets when low magnetic

Manuscript received October 5, 2004. This work was supported by the Israeli Ministry of Industry and Commerce under funds.

Y. Wolfus, A. Friedman, F. Kopansky, and Y. Yeshurun are with the Department of Physics, Institute of Superconductivity, Bar-Ilan University, Ramat-Gan 52900, Israel (e-mail: wolfuss@ @ail.biu.ac.il).

Z. Bar-Haim, Z. Ron, and N. Pundak are with RICOR-Cryogenic\&Vacuum Systems, En Harod Ihud 18960, Israel (e-mail: zvi-b@ricor.com).

Digital Object Identifier 10.1109/TASC.2005.849642

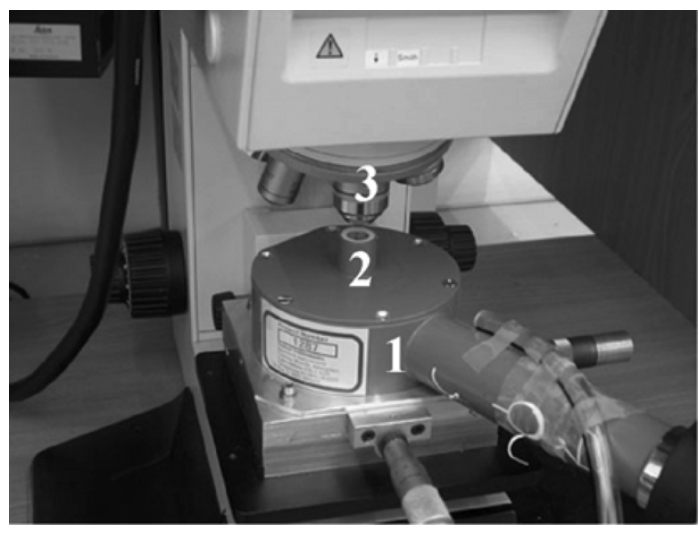

Fig. 1. Typical MO setup including a sample cryostat (1), cold pillar with optical window (2) and polarized microscope with objective wheel (3).

fields are required. Therefore, magnet design and cryogenic companies are in constant search for unique niche applications to justify the use of HTS magnet with the present tapes costs.

In this work we describe such a niche application where HTS magnets provide a preferable solution. In MO setups, the need for small working distances between the objective lenses and the inspected samples limit the volume available for external magnetic field generation coils. In this application, the volume is too restricted to allow for an economic LTS solution. At the same time, the limited volume forces high current densities, making it difficult for normal conductors to perform. HTS magnets provide a better solution for this demand.

We have designed, built and tested a compact cryogen-free HTS-magnet that complies with the magnetic field specifications required by a $\mathrm{MO}$ research setup and fits into the limited volume of this system. The design is based on three single pancakes coil and a two-stage cryocooler without radiation shield. While the required field for magneto-optical experiments is $0.1-0.2 \mathrm{~T}$ due to the saturation of the indicator, the magnet described here is capable of generating $0.35 \mathrm{~T}$ field in constant mode operation and $0.4 \mathrm{~T}$ in short time operation. A unique coil design enables fast ramping rates of the field, namely 7.4 $\mathrm{T} / \mathrm{sec}$ with $20 \mathrm{~V}$ charging voltage. The magnet is mechanically decoupled from the sample cryostat to avoid vibrations.

\section{SYSTEM REQUIREMENTS}

Fig. 1 exhibits a typical MO setup. The MO system is now a common instrument for studying the $2 \mathrm{D}$ magnetic field distribution of magnetic materials under external magnetic field [7]. A small sample cryostat (1) provides a base temperature for the inspected sample mounted on a cold pillar (2) underneath a MO indicator close to an optical window. The objective lens 
TABLE I

SUMMARY OF THE BASIC MAGNET SPECIFICATIONS REQUIRED FOR THE MO SETUP OF FIG. 1

\begin{tabular}{ll}
\hline \hline Magnetic field & $0.15 \mathrm{~T}$ \\
Operation mode & Continuous \\
Ramp rate & $2.5 \mathrm{~T} / \mathrm{sec}$ \\
External dimensions & \\
\multicolumn{1}{l}{ Height } & $30 \mathrm{~mm}$ \\
$\quad$ Inner diameter & $30 \mathrm{~mm}$ \\
Outer diameter & $200 \mathrm{~mm}$ \\
Cooling method & Cryogen-free \\
Mechanical coupling with the & No \\
sample space & \\
\hline
\end{tabular}

(3) observes the light reflected from the indicator having a polarization angle proportional to the local magnetic field inside the sample. The small working distances between the objective lenses and the inspected samples in MO setups and the need to change lenses in the objective wheel, limit the volume available for external magnetic field generation coils.

In the setup shown in Fig. 1, the height of the coil must not exceed $30 \mathrm{~mm}$. The inner room temperature bore diameter is also limited to $30 \mathrm{~mm}$. Air or water-cooled copper coils are commonly used for field generation, however these coils usually make it possible to attain limited fields of the order of 10-50 mT, in continuous operating mode because of their relatively low current densities and the joule heating that develops in the coil. The desired magnetic field value is in the range of $0.1-0.2 \mathrm{~T}$ as most of the used MO indicators saturate in this field range. To enable fast measurements of the magnetic dynamics inside the inspected samples [8], high ramping rates of the external field are required, enforcing low coil inductance and high current density. Practically, for video rate data recording, a field of $0.1 \mathrm{~T}$ should be ramped up or down within one video frame i.e. with a $2.5 \mathrm{~T} / \mathrm{sec}$ rate. Cryogen-free magnets are favorable for the field generation task because of the need to remove the coil for handling the sample. Table I summarizes the required performances of a magnet for a typical MO setup described here.

Three possible approaches were examined: Normal conductors, Low temperature superconductors (LTS) and HTS. The continuous $0.15 \mathrm{~T}$ field along with the ramp rate requirements rule out the normal conductor solution. The limited volume makes the LTS option too complicated and a costly solution. HTS coils offer a unique and cost effective solution in this case.

\section{MAGNET DESIGN}

Fig. 2 depicts the structure of the magnet system. Main components of the magnet system are the HTS coil (A), the cryostat (B) and the cryocooler (C). The magnet is built around Ricor's Coolstar 6/30 two-stage cold head. The cryostat is shaped in a reversed " $\mathrm{L}$ " shape in a way that the coil is off axis with the cryocooler axis. This way the coil is mechanically supported by the cold head, completely decoupled from the MO system to avoid mechanical vibrations. The current path includes vacuum feedthrough (1), brass current leads (2), cooled by the first stage of the cryocooler, HTS current leads (3), cooled by the first stage on one end and by the second stage on the other end, and two copper plates (4), attached to the second stage of the cryocooler. These plates have three functions: mechanical support to the

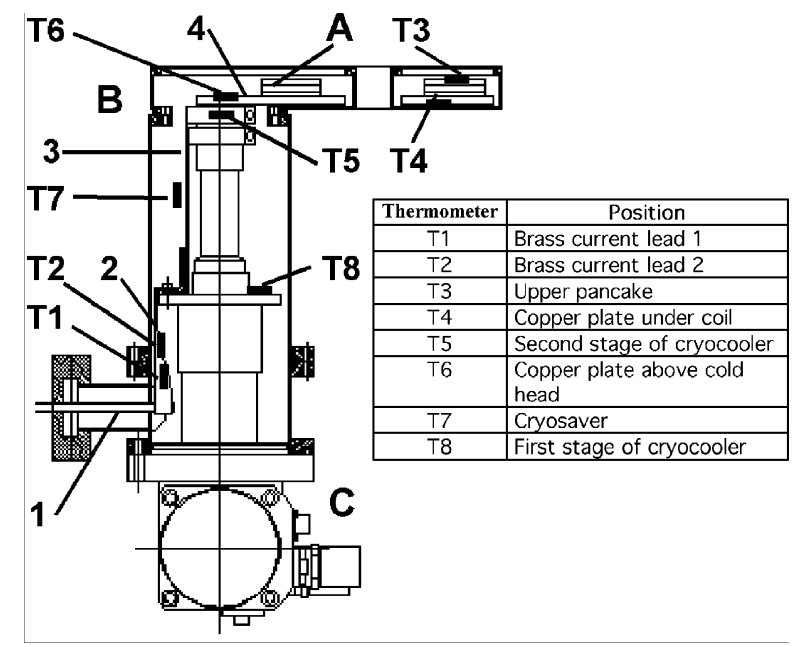

Fig. 2. Schematic structure of the magnet. (A) HTS coil, (B), Cryostat, (C) cryocooler, (1) vacuum current feedthrough, (2), brass current leads, (3) HTS current leads, T1-T8-thermometers.

HTS coil, heat conductors transferring heat from the coil to the cold head and current conductors. Three, parallel connected, CryoBlock Bi-2223 tapes by AMSC [1] form the current leads used in this magnet. These leads are connected with a flexible copper braid to copper plates attached to the first cooling stage. Brass current leads are soldered to other ends of these plates.

All conducting parts are insulated from the cryocooler by Kapton tape. Thermometers were mounted on all elements to study the thermal behavior of the system during cooling, heating and various operation conditions. Thermometers locations are marked and enumerated in Fig. 2 as T1-8. The recommended inner diameter of HTS coils is $70 \mathrm{~mm}$ according to the wire manufacturer [1]. This minimal diameter forces a use of most of the free height inside the cryostat for the coil eliminating a possible use of a radiation shield. Therefore, the coil is made of three, $33 \mathrm{~m}$ long single pancakes, with 100 turns each. We have used AMSC high-Strength Wire [1] with a nominal self-field critical current of $125 \mathrm{~A}$ at $77 \mathrm{~K}$. The lack of radiation shield makes it difficult to foresee the equilibrium temperatures of all system components. Therefore, the operating current for achieving the required $0.1-0.2 \mathrm{~T}$ was set to $40 \mathrm{~A}$ to ensure safety margins for coil operation in temperatures as high as $77 \mathrm{~K}$. The Magnetic field of the coil was calculated by FEA (Vector Fields) software. The magnetic field distribution (axial component in the central zone, radial component on the coil turns and field lines) is shown in Fig. 3.

For 40 A coil current, a field intensity of $0.14 \mathrm{~T}$ is obtained both for the axial field in the MO inspected sample location and for the radial field at the winding location of the end pancakes. The latter component is the field component perpendicular to the tapes broad face limiting the critical current. Prior to winding the coil, we applied a numerical technique for estimating the critical current of the coil under self-field conditions [9]. The simulation suggested that the coil current density would fall to about $40 \mathrm{~A}$ at $77 \mathrm{~K}$, given a self-perpendicular field of $0.14 \mathrm{~T}$.

Fig. 4 exhibits the I-V characteristics of the 3 single individual pancakes and the integrated coil when immersed in LN. The measurements confirm the numerical analysis. While the 


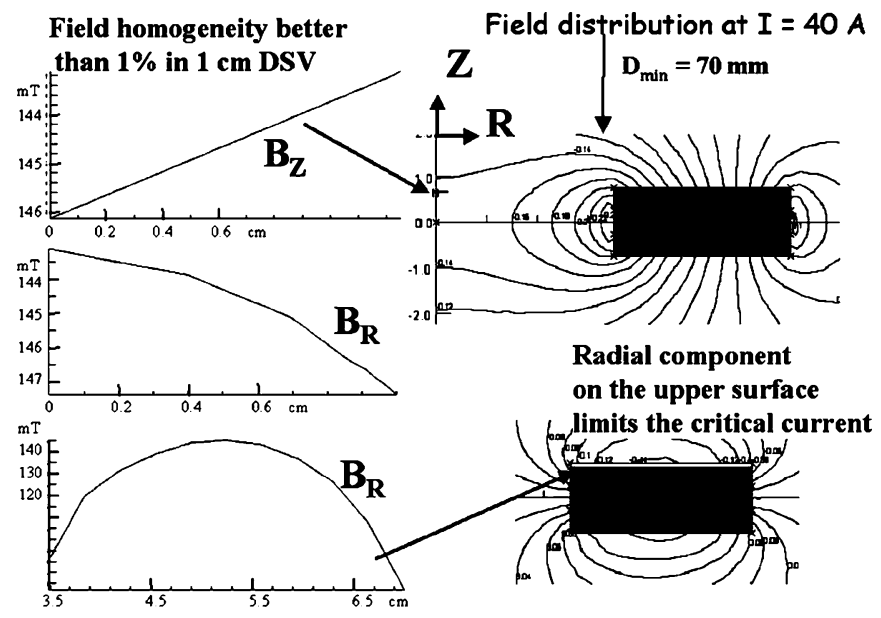

Fig. 3. Magnetic field distribution calculated for the HTS coil. Top to bottom: axial field lines of the coil, dependence of the axial field component along the coil main axis, radial dependence of the axial field component, radial field lines in the coil and its vicinity, and radial field distribution at the edge of the coil.

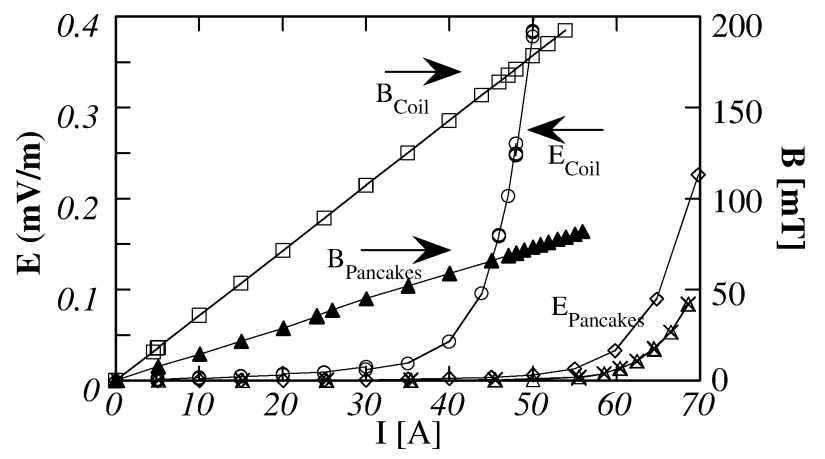

Fig. 4. Left axis: $\mathrm{I}-\mathrm{V}$ curves of individual single pancakes (empty triangles, $\mathrm{x}$ and diamonds) and of the integrated coil (circles). Right axis: I-B curves of the individual pancakes (full triangles) and the integrated coil (squares).

individual pancakes show a critical current value of $62 \pm 3 \mathrm{~A}$, the critical current drops to $42 \mathrm{~A}$ in the integrated coil. As expected, this is a result of the strong increase in perpendicular field at the top and bottom pancakes. The magnetic field generated by the coil in these conditions is about $0.15 \mathrm{~T}$. The results obtained at $77 \mathrm{~K}$ leave large enough margins for cryogen-free operation.

One of the required magnet specifications is a field ramp rate of $2.5 \mathrm{~T} / \mathrm{sec}$. To cope with this requirement, the design includes minimal metal components in the coil vicinity. The only metal coil support is the copper plate (marked as 4 in Fig. 2) at the coil bottom serving as current lead and thermal anchoring. This plate is cut to reduce eddy currents during a fast field ramp. In addition, a unique, wet winding method has been developed and applied for the pancakes winding. This new technique ensures higher coil current densities and thermal conductivity than commonly used winding methods. The technique is patent pending and will be described elsewhere.

\section{MAGNET OPERATION}

Fig. 5 shows the recorded temperature in various locations within the magnet during an initial cool down. The system fully stabilizes after 5 hours. At this time the coil temperature is close

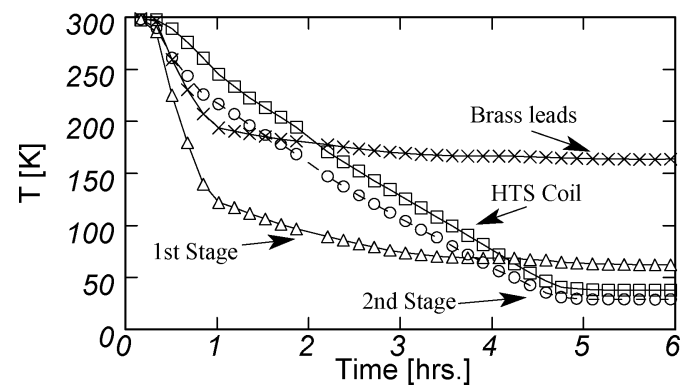

Fig. 5. Time dependent temperature in various cryostat locations during the initial cooling down process.

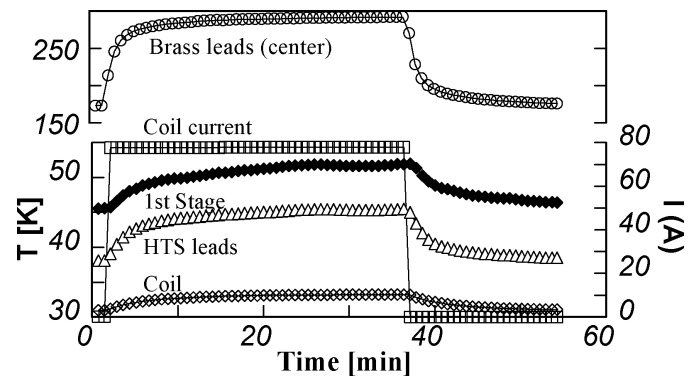

Fig. 6. Time dependent temperature in various cryostat locations for continuous operations at $80 \mathrm{~A}$.

to $40 \mathrm{~K}$ and the cryostat base temperature obtained at the 2-nd cooling stage is about $30 \mathrm{~K}$. The 1-st cooling stage stabilizes at about $62 \mathrm{~K}$, ensuring a smooth operation of the HTS leads. The figure also demonstrates that the magnet crosses the $77 \mathrm{~K}$ line after about $3.5 \mathrm{~h}$. At this time the magnet is ready for basic mode operation. The initial cooling time presented here is a result of the compact design and is acceptable by the operators of the MO system.

Fig. 6 demonstrates the time dependent temperature after applying $80 \mathrm{~A}$ current, twice the designed value, in a constant current operation mode. Note that the base temperatures of all system components are lower than in the initial cool down (Fig. 5). This is a result of magnet degassing after a few temperature cycles of the magnet. Upon applying 80 A current, the brass leads develop high Joule heating (central point of the leads is shown in the upper curve of Fig. 6), transfer heat into the cold head and cause a general temperature increase in all magnet components. However, this heat flow into the magnet is mostly absorbed by the 1-st cooling stage with a shift of its base temperature from $45 \mathrm{~K}$ to $53 \mathrm{~K}$. The mid point of the HTS leads also rises by $8 \mathrm{~K}$. Further heat absorption in the 2-nd cooling stage limits the temperature rise in the coil to about $3 \mathrm{~K}$. Since the magnet reaches a steady state after more than 30 min of continuous operation in 80 A current, we conclude that the built magnet system is capable of generating $0.3 \mathrm{~T}$ field in continuous mode operation.

Further tests of the magnet include shutting down the cryocooler while a 50 A current continues to run through the coil. Fig. 7 displays the temperature variation when the cryocooler is stopped for $5 \mathrm{~min}$. The 1-st cooling stage heats substantially causing other cold components to heat. Approximately $15 \mathrm{~min}$ after the cryocooler is turned on again, the magnet returns to thermal equilibrium. 


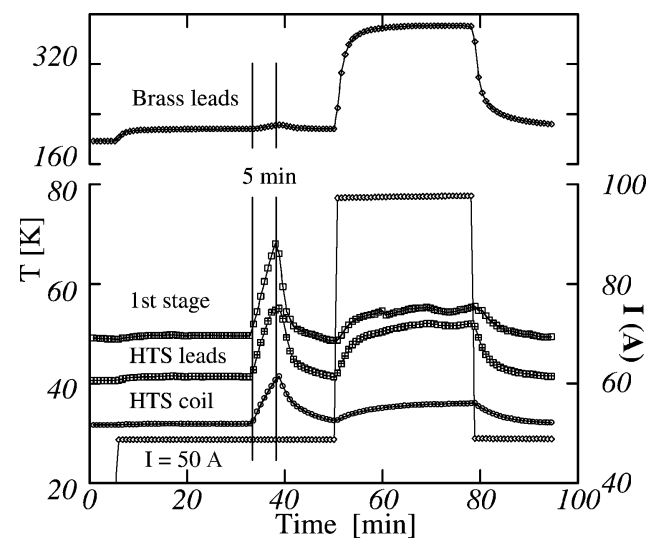

Fig. 7. Time recorded temperature in the magnet when carrying $50 \mathrm{~A}$ and 100 A currents. In the first case, the cryocooler was shut down for $5 \mathrm{~min}$.

During all this time of severe operation conditions, the magnet continues to operate with no interruptions. The figure also shows that after returning to equilibrium the magnet was tested with 100 A current for $30 \mathrm{~min}$. While the cold components are still safe, the heating of the brass leads is evident. Out of precaution, the current was not raised beyond the $100 \mathrm{~A}$ value, however it is clear that a reduction of the brass leads resistance would allow for higher currents/fields in this same magnet.

Finally, the magnet was tested for its ramp rates. An AC power supply was connected to the current terminals. A $20 \mathrm{~V}$ charging voltage pulse, with frequency of $8 \mathrm{~Hz}$ was applied to the magnet and the resulting current and magnetic field were registered. Negligible phase shift between the current and the field has been observed, implying that eddy currents are also negligible. The ramp rate extracted from the slope of the field curve is $7.4 \mathrm{~T} / \mathrm{sec}$. To achieve higher ramp rates, a power supply with a higher voltage is required.

\section{SUMMARY}

We have designed, built and tested a compact cryogen-free HTS magnet to comply with the special volume and field re- quirements of a magneto-optical system. The magnet presented here is based on a 3-pancakes coil and a compact cryostat built around a 2-stage cryocooler. The magnet achieves $0.4 \mathrm{~T}$ field and ramp rate of $7.4 \mathrm{~T} / \mathrm{sec}$, well above the requirements of the MO setup.

The success of the project described in this work may serve as an example for possible use of HTS cryogen-free magnets. In cases of limited space, where moderate magnetic fields are required, HTS may provide a preferable solution.

\section{ACKNOWLEDGMENT}

The authors would like to thank S. Natliashvili for the assistance in the coil design, A. Santo, and S. Asulay for the assistance in the tests.

This study was carried out in the framework of a joint collaborative project of Bar-Ilan University and RICOR-Cryogenic\&Vacuum Systems.

\section{REFERENCES}

[1] http://www.amsuper.com [Online]

[2] http://trithor.de/gfx/spec_standard.pdf [Online]

[3] http://www.sumitomoelectricusa.com/scripts/products/scc/wire.cfm [Online]

[4] K. Tasaki, T. Kuriyama, S. Nomura, Y. Sumiyoshi, H. Hayashi, H. Kumura, K. Tsusumi, M. Iwakuma, and K. Funaki, "Thermal stability of a cryocooler-cooled HTS pancake coil wound with Bi2223 tape," Physica $C$, vol. 392-396, pp. 1210-1213, 2003.

[5] H. Kasahara, S. Akita, K. Tasaki, A. Tomioka, T. Hase, K. Ohata, N. Ohtani, and H. Sakaguchi, "Basic characteristic evaluation of cryocooler-cooled HTS coils," IEEE Trans. Appl. Supercond., vol. 12, pp. 766-769, 2002.

[6] K. Tasaki, M. Ono, and T. Kuriyama, "Study on AC losses of a conductive cooled HTS coil," IEEE Trans. Appl. Supercond., vol. 13, pp. 1565-1568, 2003.

[7] T. H. Johansen and D. V. Shantsev, Eds., Magneto-Optical Imaging. Dordrecht: Kluwer, 2004, p. 345.

[8] B. Kalisky, A. Shaulov, T. Tamegai, and Y. Yeshurun, "Dynamics of transient disordered states in $\mathrm{Bi}_{2} \mathrm{Sr}_{2} \mathrm{CaCuO}_{8+\mathrm{d}}$, Phys. Rev. B, vol. 68, pp. 24 515-24 520, 2003.

[9] Y. Wolfus, Y. Fleger, A. Friedman, F. Kopansky, B. Kalisky, Y. Yeshurun, Z. Bar-Haim, Z. Ron, L. Ying, and N. Pundak, "Estimation of critical current of BSCCO tapes," Physica C, vol. 401, pp. 222-226, 200. 